\title{
Simulation of motorcyclist's kinematics during impact with W-Beam guardrail
}

\begin{abstract}
W-Beam guardrail system has been in use as a standard for roadside safety barrier since 1950s. Recently, its safety performance standard has been upgraded to absorb impact from large vehicles. This performance standard requires guardrail system to be capable of capturing and redirecting a large range of vehicle types and sizes but its effects on safety of motorcyclists are not yet understood. The paper describes a three-dimensional computer simulation of the kinematics impact of motorcycle and dummy rider with W-Beam guardrail inclined at angles 45 and $90^{\circ}$ to the initial direction of travel. The simulation is based on the test procedure recommended by ISO 13232 on the configurations for motorcycle-car impact. The focus of this study is not on the motorcycle change in velocity, but on the rider's kinematics and acceleration vs. time history. Multibody model of motorcycle and finite element model of guardrail were developed in commercially available software. The simulation results are presented in this paper in form of kinematics and acceleration vs. time history.
\end{abstract}

Keyword: Kinematics, Impact, Acceleration-time history, Motorcycle, Guardrail 